\title{
Improving the Life Cycle Performance of Chemical Products and Materials Through Data Exchange Along the Value Chain-Synthesis of LCM2017 Session Presentations
}

\author{
Guido Sonnemann and Carmen Alvarado
}

\begin{abstract}
This paper provides a summary of what has been presented and discussed at the 8th international conference on Life Cycle Management (LCM 2017 conference, Luxembourg), during the session entitled "Improving the life cycle performance of chemical products and materials through data exchange along the value chain". The purpose of the session was to demonstrate how to assess and manage the global sustainability of chemical products and materials, taking into account their whole life cycle, to achieve real improvements. Over the six oral presentations, the most challenging issues regarding availability and transparency of Life Cycle Inventory data from the chemicals industry were discussed. Some approaches developed in order to face these challenges were detailed, illustrated also with the presentation of some specific case-studies.
\end{abstract}

\section{Introduction}

Most companies are part of long and sometimes complex supply chains. Often the control over the environmental impacts along the value chain is limited for one single company. The purpose of the session was to demonstrate how to assess and manage the global sustainability of chemical products and materials, taking into account their whole life cycle, to achieve real improvements. This includes the identification of hot spots and work towards sustainable innovation. Radical improvement along the value chain is only possible with close collaboration with

\author{
G. Sonnemann $(\square)$ \\ University of Bordeaux, CNRS, UMR 5255, 33405 Talence Cedex, France \\ e-mail: guido.sonnemann@u-bordeaux.fr \\ C. Alvarado \\ Akzo Nobel Chemicals, 3818 LE Amersfoort, The Netherlands \\ G. Sonnemann \\ CNRS, ISM, UMR 5255, 33405 Talence Cedex, France \\ (C) The Author(s) 2018 \\ E. Benetto et al. (eds.), Designing Sustainable Technologies, \\ Products and Policies, https://doi.org/10.1007/978-3-319-66981-6_11
}


suppliers, for example, through sharing supplier specific data on environmental impacts of raw materials. There are many obstacles which need to be overcome before sharing data along the value chain: trust, data formats, methodology harmonization, etc. The PEF (Product Environmental Footprint), together with Sustainability and the WBCSD life cycle metrics for chemical products are good examples of platforms that can help companies share information in a safe, fair and efficient way to steer to real improvements towards sustainable development. Six presentations were selected for the session. The main outcomes of each of them are summarised in this paper.

\section{Rapid Estimation of Life Cycle Inventories}

Many regulations and company sustainability activities are based on the application of Life Cycle Assessment. A significant challenge is the amount of data that needs to be compiled, harmonized and modelled before obtaining meaningful and robust results.

In this context, the United States Environmental Protection Agency (U.S. EPA) developed an approach to rapidly generate more comprehensive and reliable life cycle inventories (LCI) than those currently available. The basic approach to generating such inventories is to combine four individual efforts:

(1) top-down data mining,

(2) bottom-up simulation (combining material and energy balances with emissions modelling),

(3) LCI chemical lineage (computationally tracing the ancestry of a chemical alternative), and

(4) LCI reconciliation of both the top-down and bottom-up approaches.

Life Cycle Inventories developed through this approach can be updated once new datasets are available [1].

\section{Current Models and Approaches for Data Matching and Modelling of Chemical Production}

Ecoinvent is a data provider for the Product Environmental Footprint (PEF) pilot phase, driven by the European Commission. In this context many of the data tenders are based on ecoinvent LCI data in the background and ecoinvent was selected as the direct data supplier for 270 products and over 800 datasets, on the production of chemicals. The challenge was to deal with significant data needs on chemical products on short notice (3 months), while delivering quality and integrating primary data. 
Around 80 chemicals needed completely new datasets. Around 200 new chemicals unit process datasets were created. The industry stakeholders group discussion involved in the PEF pilot phase was organised jointly with the creation of these datasets. The short timeframe made the collaboration difficult, but several sources provided data. A framework model that provides a unit process result, was created in a way that each part of the process is adaptable. Thanks to the "modular model" approach chosen, the datasets can be upgraded on specific topics when data becomes available. A stoichiometric model of the reactions was required, with reactants and other reagents. Average values for yield, heat electricity, water and supporting processes were available from industry reports. The air emission model was based on vapour pressure, filtering systems and toxicity. The waste water treatment model used determines the water emissions. Previous models existed but all aspects of the new model have been updated and extended for this project.

The project was an interesting combination of data format and data requirement issues and high efficiency data generation. The flexible model of chemical unit processes allows users to work with various data availabilities. The model is accessible, with all values and sources. The datasets list source types and acknowledge industry sources in coordination with the data providers. The data are now used in the PEF pilot projects, and many will also be published in the upcoming ecoinvent 3.4 [2].

\section{PlasticsEurope Experience in Managing Information Along the Value Chain for RA and LCA}

Risk assessments are carried out by PlasticsEurope members for each substance. It takes the form of lists of substances with maximum limit of concentration or migration. The full chemical composition of the plastics must be known, including traces of starting substances, additives, impurities at very low thresholds. The information must be transmitted in the supply chain so that the one putting the packed product on the market has the data on the plastics available. When there is no sectorial organisation, each actor interprets differently the requirements of information and sends questionnaires upstream. They are all different and tend to ask more than required. Suppliers hesitates to send information downstream since they are afraid of misinterpretation and misuse due to the fear of being challenged (substance ban, deselection...). There is a need for a common playing field in the supply chain with a common interpretation of regulation, mutual understanding, respect of confidentiality and trust.

The situation for life cycle inventory data is similar: confidentiality issues protection of competitiveness and know-how, competition law with a large grey zone of interpretation, fear of giving readability on costs and of being deselected, etc. That means also here it is primordial to build mutual understanding and trust. The global guidance principles on Life Cycle Assessment (LCA) databases are a good 
starting point. For the way forward an approach is proposed that differentiates averaged and individual datasets. For averaged datasets partial disaggregation is possible for commodities (large number of producers) but partial disaggregation is not possible when there are not enough producers ( 3 being an absolute minimum). For individual dataset, it is up to business to decide. Most often, it is confidential. Overall there is a need for common rules. As an example PlasticsEurope's recommendation on Steam Cracker allocation for the sake of comparability of petrochemical products was given [3].

\section{Biomass Balance Approach: An Innovative and Complementary Approach for Using Biomass in the Chemical Industry}

BASF recognises four drivers for including renewables in their portfolio: competitiveness, upcoming regulations, climate change and raw material diversification. BASF has developed a methodology to account and communicate the renewable content of his materials to customers. This approach is an intermediate situation on the way to dedicate production of materials with renewable content. The latter is often costly as the market is not matured yet.

The biomass balance approach is similar to that for "green" electricity: The fossil raw materials needed to produce a customer's products are replaced by renewable raw materials. Only the quantities of renewable raw materials requested by a customer are purchased up front by BASF and managed in the SAP system. When a customer places an order, the required quantities of renewable feedstock are allocated to the products purchased.

The advantages of this approach are the production of drop-in products, easy accountability and it can be implemented directly [4].

\section{Sustainability at Teijin: Customer Benefit Model and Case Study Tires}

Being eco-effective is important for Teijin and for this reason eco-efficiency assessments are often made to their products. With the help of this assessment Teijin is able to assess the real eco-performance of its products. The presented case study was about car tires, where $90 \%$ of the impact along the value chain is in the use phase, due to consumption of fuel. Therefore, products with lower weight and less rolling resistance turn out to show a better eco-efficiency performance [5]. 


\section{Life Cycle Assessment of Acrylic Fibre and Garment in Thailand}

A case study is presented that aims first at the quantification of environmental impacts by LCA according to ISO 14040/44 standard [6, 7], for one tonne of Acrylic Fibre manufactured at Thai Acrylic Fibre Co. Ltd, i.e. cradle to gate study, and then for one piece of Acrylic Garment of $450 \mathrm{~g}$ throughout the entire life cycle, i.e. cradle to grave study with 100 washes. Acrylonitrile, electricity and steam consumption were identified as major hot-spots in the value chain across the various identified environmental impacts. Use-stage electricity and water consumption are also hot-spots in cradle to grave study. This information is used for the development of a report for communication to stakeholders. Improvements are possible in the captive power plant efficiency, the steam and electricity consumption in polymerisation, the solvent recovery and the wet-spinning processes. Scenarios of different usages of acrylonitrile produced through the propane route will be analysed in the future to further understand the processes and improve them [8].

\section{Conclusions}

The presentations provided a good overview of how Life Cycle Assessment is used to assess chemical products and materials and which challenges exist with regard to the data exchange along the value chain. The session shows how companies use LCA results to improve the chemical products in their journey towards sustainability, in which they use life cycle management. Some presentations also gave an insight in the progress made in the quality and the possibilities of preparing LCIs of chemical products and materials.

Overall, including also poster presentations, it can be said that the chemicals industry has achieved an important degree of maturity in implementing life cycle management since the beginning of this century. In the future it can be expected that the data exchange along the value chain will be further improved, using as an example the established exchange of toxicity information stemming from risk assessment.

\section{References}

1. Smith R-L, Meyer D-E, Ruiz-Mercado G-J, Gonzalez M-A, Abraham J-P, Barrett W-M, Randall P-M, Niblick B, Mittal V-K, Rapid Estimation of Life Cycle Inventory, LCM 2017 conference, Luxembourg, 2017.

2. Wernet G, Levova T, Bourgault G, Current models and approaches for data matching and modeling of chemical production, LCM 2017 conference, Luxembourg, 2017. 
3. Castelan G, De Hults Q, PlasticsEurope experience in managing information along the value chain for RA and LCA, LCM 2017 conference, Luxembourg, 2017.

4. Krüger C, Kicherer A, Kormann C, Raupp N, Biomass Balance: an innovative and complementary approach for using biomass in the chemicals industry, LCM 2017 conference, Luxembourg, 2017.

5. Beers H, Bosman H, Kamer M, Huguet Ferran P, Sustainability at Teijin: Customer Benefit Model and Case Study Tires, LCM 2017 conference, Luxembourg, 2017.

6. ISO 14040: Environmental management-Life cycle assessment-Principles and framework, Geneva, 2006.

7. ISO 14044: Environmental management—Life cycle assessment—Requirements and guidelines, Geneva, 2006.

8. Barik J, Singh R, Life Cycle Assessment of Acrylic Fiber and Garment in Thailand, LCM 2017 conference, Luxembourg, 2017.

Open Access This chapter is licensed under the terms of the Creative Commons Attribution 4.0 International License (http://creativecommons.org/licenses/by/4.0/), which permits use, sharing, adaptation, distribution and reproduction in any medium or format, as long as you give appropriate credit to the original author(s) and the source, provide a link to the Creative Commons license and indicate if changes were made.

The images or other third party material in this chapter are included in the chapter's Creative Commons license, unless indicated otherwise in a credit line to the material. If material is not included in the chapter's Creative Commons license and your intended use is not permitted by statutory regulation or exceeds the permitted use, you will need to obtain permission directly from the copyright holder.

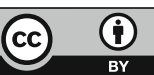

